\title{
Jian-Pi-Yi-Shen Formula ameliorates chronic kidney disease: involvement of mitochondrial quality control network
}

\author{
Xinhui Liu ${ }^{1 \dagger}$, Jianping Chen ${ }^{2 \dagger}$, Xiaoyan Liu ${ }^{1,2}$, Dongtao Wang ${ }^{1}$, Ping Zheng ${ }^{2}$, Airong Qi ${ }^{1}$, Tiegang $\mathrm{Yi}^{1,2^{*}}$ and
} Shunmin $\mathrm{Li}^{1 *}$

\begin{abstract}
Background: Jian-Pi-Yi-Shen Formula (JPYSF), a Chinese herbal decoction with the efficacies of 'fortify the spleen and tonify the kidney' and 'activate blood and resolve stasis', is effective for the treatment of chronic kidney disease in clinic. However, the underlying mechanism remains unclear. The aim of this study was to investigate the therapeutic effects and possible mechanisms of JPYSF on retarding chronic kidney disease progression in 5/6 nephrectomized $(5 / 6 \mathrm{Nx})$ rats.

Methods: Perindopril (4 mg/kg/d) and JPYSF $(2.72 \mathrm{~g} / \mathrm{kg} / \mathrm{d})$ were administrated by gavage to $5 / 6 \mathrm{Nx}$ rats daily for 6 weeks. The therapeutic effects of JPYSF were evaluated by renal function, pathological injury, and fibrosis. The protein levels associated with mitochondrial quality control network were measured by Western blot and immunofluorescence analysis.

Results: 5/6 Nx rats showed obvious decline in renal function as evidenced by increased serum creatinine, blood urea nitrogen, and urinary protein excretion, and significant injury in kidney structure as evidenced by glomerular hypertrophy, tubular atrophy, and interstitial fibrosis. Administration of JPYSF for 6 weeks could improve renal function and ameliorate kidney structure injury in 5/6 Nx rats. Furthermore, the remnant kidneys of 5/6 Nx rats showed unbalanced mitochondrial quality control network manifested as decreased mitochondrial biogenesis, fusion, and mitophagy, and increased mitochondrial fission. Treatment of JPYSF could restore aforesaid aspects of mitochondrial quality control network.
\end{abstract}

Conclusions: These results indicate that JPYSF can notably ameliorate 5/6 Nx-induced chronic kidney disease, which may be related with modulation of mitochondrial quality control network.

Keywords: Traditional Chinese medicine, Chronic kidney disease, Fibrosis, Jian-Pi-Yi-Shen formula, Mitochondrial quality control network

\section{Background}

Chronic kidney disease (CKD) is a common chronic disease with an estimated global prevalence of approximately $8-16 \%$ [1]. Despite this, there are relatively few therapies in developing for the treatment of CKD. For patients with CKD, the role of the renin-angiotensin system modulation only exerts partial salutary effects and

\footnotetext{
*Correspondence: tg.yi@126.com; zyylishunmin@163.com

${ }^{+}$Xinhui Liu and Jianping Chen contributed equally to this work.

'Department of Nephrology, Shenzhen Traditional Chinese Medicine

Hospital, Guangzhou University of Chinese Medicine, Shenzhen, Guangdong, China

Full list of author information is available at the end of the article
}

can not necessarily prevent the progression to end-stage renal disease and the need for renal replacement therapy $[2,3]$. The limit option for CKD treatment has prompted patients to seek out some alternative strategies such as traditional Chinese medicines (TCM) [4-8]. The prevalence of CKD in China is $10.8 \%$ [9], and TCM is widely used for CKD treatment in China [10-13]. However, the question of TCM for CKD patients remains a matter of debate. A recent study provided solid evidence of the beneficial effects of prescribed TCM on CKD patients in Taiwan [14] supporting that TCM can be an attractive area for the development of therapeutic drugs on CKD.

(c) The Author(s). 2018 Open Access This article is distributed under the terms of the Creative Commons Attribution 4.0 International License (http://creativecommons.org/licenses/by/4.0/), which permits unrestricted use, distribution, and reproduction in any medium, provided you give appropriate credit to the original author(s) and the source, provide a link to the Creative Commons license, and indicate if changes were made. The Creative Commons Public Domain Dedication waiver (http://creativecommons.org/publicdomain/zero/1.0/) applies to the data made available in this article, unless otherwise stated. 
Jian-Pi-Yi-Shen Formula (JPYSF), a Chinese herbal decoction, is composed of eight herbs, that is Astragali Radix, Atractylodis Macrocephalae Rhizoma, Dioscoreae Rhizoma, Cistanches Herba, Amomi Fructus Rotundus, Salviae Miltiorrhizae Radix et Rhizoma, Rhei Radix et Rhizoma, and Glycyrrhizae Radix et Rhizoma Praeparata cum Melle. According to TCM theory, JPYSF possesses the efficacies of 'fortify the spleen and tonify the kidney' and 'activate blood and resolve stasis' [15]. JPYSF was combined and modified from two traditional herbal decoctions namely Da-Huang-Gan-Cao-Tang (DHGCT) and Yu-Ping-Feng-San (YPFS). DHGCT was recorded in Jin Gui Yao Lue by Zhongjing Zhang (150 B.C. to A.D. $219)$, which is considered to remove static blood or excessive fluid through the bowels. YPFS was described in Dan Xi Xin Fa by Danxi Zhu in Yuan Dynasty (A.D. 1279-1368), which is being used to replenish "Qi". To induce purgation by DHGCT and to replenish "Qi" by YPFS could be applied for the treatment of CKD-associated urine toxins retention and low immune response. For over 20 years, JPYSF has been clinically prescribed as basic formula for the treatment of patients with CKD. Results of our previous clinical study suggested that JPYSF significantly improved kidney function of CKD especially mild-to-moderate CKD patients, as evidenced by reducing serum creatinine (Scr) and blood urea nitrogen (BUN) levels $[16,17]$. However, the underlying action mechanism of JPYSF remains unidentified and needs to be investigated.

The kidney is a highly aerobic organ and is rich in mitochondria. Therefore, kidneys are exquisitely dependent upon, and susceptible to, being damaged by mitochondria [18]. Studies have shown significantly increased reactive oxygen species production and abnormal respiratory chain complex expression in peripheral blood mononuclear cells of CKD patients, thereby demonstrating the closely association between mitochondrial dysfunction and CKD [19, 20]. Recent studies have also demonstrated that mitochondria participate in CKD progression and mitochondrial dysfunction led to increased proteinuria [21, 22], uremic toxin retention [23, 24], NLRP3 inflammasome activation [25] and transforming growth factor- $\beta$ expression [26]. Healthy mitochondria are essential for kidney and they are maintained by a mitochondrial quality control network including mitochondrial biogenesis, mitochondrial fission and fusion and mitochondrial autophagy (mitophagy) [27]. However, the alteration of mitochondrial quality control network in CKD is still unclear. In the present study, we hypothesized that abnormal mitochondrial quality control network occurs in CKD development, and JPYSF treatment could restore mitochondrial quality control network in rat with $5 / 6$ nephrectomy $(5 / 6 \mathrm{Nx})$. Perindopril is a commonly used angiotensin converting enzyme inhibitor in the treatment of CKD. Therefore, we included a positive control group treated by perindopril to evaluate the effect of JPYSF on improving kidney function and structure in 5/6 Nx rats. And we further investigated the role of JPYSF in modulating mitochondrial quality control network.

\section{Methods}

\section{Chemicals and antibodies}

Sodium danshensu (1), salvianolic acid B (2), echinacoside (3), liquiritin (4), acteoside (5), calycosin 7-O- $\beta$-glucoside (6), astragaloside IV (7), formononetin (8), and hesperidin (internal standard, ISTD) were purchased from National Institutes for Food and Drug Control (Beijing, China). The purity of all marker chemicals were determined to be no less than $98 \%$ by normalization of peak areas, as revealed by HPLC-DAD. HPLC grade acetonitrile was purchased from Merck (Darmstadt, Germany), and ultrapure water was prepared using a Milli-Q purification system (Molsheim, France). Other reagents used here were of analytical grade. Perindopril was purchased from Sigma-Aldrich (St Louis, MO, USA). The primary antibodies included rabbit anti-peroxisome proliferator-activated receptor- $\gamma$ coactivator-1 $\alpha$ (PGC-1 $\alpha)$, rabbit anti-PTEN-induced putative kinase 1 (PINK1) (Novus, Littleton, CO, USA), mouse anti-mitochondrial transcription factor A (TFAM), rabbit anti-nuclear respiratory factor 1 (NRF-1) (Santa Cruz Biotechnology, Santa Cruz, CA, USA), rabbit anti-dynaminrelated protein 1 (Drp-1), rabbit anti-mitofusin 2 (Mfn-2), rabbit anti-heat shock protein-60 (HSP-60), rabbit anti-cytochrome $c$ oxidase subunit IV (COX-IV), mouse anti- $\alpha$-tubulin (Cell Signaling Technology, Beverly, MA, USA), mouse anti-optic atrophy 1 (OPA-1) (BD Biosciences, San Jose, CA, USA), rabbit anti-Parkin (phospho S65), rabbit anti-fibronectin, rabbit anti-type IV collagen, rabbit anti-lysosomal-associated membrane protein 1 (LAMP-1), mouse anti-cytochrome c oxidase subunit I (COX-I), mouse anti-nicotinamide adenine dinucleotide dehydrogenase (ubiquinone)- $1 \beta$ subcomplex 8 (NDUF $\beta 8$ ) (abcam, Cambridge, MA, USA), mouse anti-Parkin, mouse anti- $\alpha$-smooth muscle actin ( $\alpha$-SMA) (Sigma-Aldrich, St Louis, MO, USA), rabbit anti-ATP synthase subunit beta (ATP5B) (Aviva Systems Biology, San Diego, CA, USA), and mouse anti-glyceraldehyde-3-phosphate dehydrogenase (GAPDH) (proteintech, Wuhan, China). Horseradish peroxidase (HRP)-conjugated anti-mouse IgG and HRP-conjugated anti-rabbit IgG were purchased from Life Technologies (Carlsbad, CA, USA).

\section{Plant materials and preparation of JPYSF water extract}

The herbal composition and proportion of JPYSF was summarized in Table 1. Raw herbs were purchased from Shenzhen Huahui Pharmaceutical Co., Ltd. (Shenzhen, China). The plant materials were authenticated by 
Table 1 The herbal composition and proportion of JPYSF

\begin{tabular}{|c|c|c|c|c|}
\hline Botanical name & Herbal name & Chinese name & Voucher number & Dosage \\
\hline $\begin{array}{l}\text { Astragalus membranaceus (Fisch.) Bge. } \\
\text { var. mongholicus (Bge.) Hsiao }\end{array}$ & Astragali Radix & Huang-Qi & $2010015 Z$ & $30 \mathrm{~g}$ \\
\hline Atractylodes macrocephala Koidz. & Atractylodis Macrocephalae Rhizoma & Bai-Zhu & $2010024 Z Z$ & $10 \mathrm{~g}$ \\
\hline Dioscorea opposita Thunb. & Dioscoreae Rhizoma & Shan-Yao & $2010037 Z$ & $30 \mathrm{~g}$ \\
\hline Cistanche deserticola Y.C. Ma & Cistanches Herba & Rou-Cong-Rong & $2040056 Z$ & $10 \mathrm{~g}$ \\
\hline Amomum kravanh Pierre ex Gagnep. & Amomi Fructus Rotundus & Dou-Kou & $202086 Z$ & $10 \mathrm{~g}$ \\
\hline Salvia miltiorrhiza Bunge. & Salviae Miltiorrhizae Radix et Rhizoma & Dan-Shen & $2010006 Z$ & $15 \mathrm{~g}$ \\
\hline Rheum palmatum L. & Rhei Radix et Rhizoma & Da-Huang & $2010040 Z$ & $10 \mathrm{~g}$ \\
\hline Glycyrrhiza uralensis Fisch. & Glycyrrhizae Radix et Rhizoma Praeparata cum Melle & Zhi-Gan-Cao & $2010008 Z Z$ & $6 \mathrm{~g}$ \\
\hline
\end{tabular}

Shangbin Zhang, the executive manager of Pharmaceutical Department, Shenzhen Traditional Chinese Medicine Hospital (STCMH) based on their morphological characteristics. The voucher specimens were kept at STCMH. Assurance of quality control for all the materials was validated according to the Chinese Pharmacopeia (China Pharmacopoeia Committee, 2015). Raw herbs were weighed and boiled twice in 8 times of $\mathrm{ddH}_{2} \mathrm{O}(w / v)$ for 1 $\mathrm{h}$ per time. The extraction liquid was centrifuged $(13,000$ rpm, $10 \mathrm{~min}$ ), the supernatant was kept at $4{ }^{\circ} \mathrm{C}$ and filtered through a $0.22 \mu \mathrm{m}$ filter (Millipore Ireland Ltd., Ireland) before HPLC analysis. For animal studies, the extract was dried by freeze dryer and stored at $-80^{\circ} \mathrm{C}$. Before the treatment, the freeze-dried powder was re-dissolved with dd $\mathrm{H}_{2} \mathrm{O}$ to get JPYSF extract.

\section{Chromatographic conditions and instrumentation}

Validation HPLC method was performed on a Shimadzu (Kyoto, Japan) LC-20AT system, which was equipped with a degasser, a binary pump, an autosampler and a diode array detector. The herbal extract was separated on Agilent ZORBAX SB-C18 $(250 \mathrm{~mm} \times 4.6 \mathrm{~mm}, 5 \mu \mathrm{m})$ column. The mobile phase was composed of acetonitrile (A) and 10 $\mathrm{mmol} / \mathrm{L}$ ammonium acetate (B) using the following gradient program: 0-1.2 min, 5\% A; 1.2-2 min, 5-20\% A; 2-4 min, 20-40\% A; 4-8 min, 40\% A; 8-10 min, 40-95\% A; 10-17 $\mathrm{min}, 95 \% \mathrm{~A} ; 17-20 \mathrm{~min}, 5 \% \mathrm{~A}$; the flow rate was 0.8 $\mathrm{ml} / \mathrm{min}$; the injection volume was $5 \mu \mathrm{L}$. A Shimadzu mass spectrum (LC-2020) equipped with an ESI ion source was operated in positive and negative modes, and the selected ion monitoring was used. The drying gas temperature was $350{ }^{\circ} \mathrm{C}$; drying gas flow: $1.5 \mathrm{~L} / \mathrm{min}$; nebulizer pressure: 35 psi; capillary voltage: $3500 \mathrm{~V}$. Shimadzu Mass workstation software was used for data acquisition and processing.

\section{Animals and experimental treatment}

All animal experiments were conducted with protocols approved by the Ethics Committee of Guangzhou University of Chinese Medicine and in accordance with $\mathrm{Na}$ tional Institutes of Health Guideline for the care and use of laboratory animals (NIH Publications No. 80-23, revised 1996). Eight weeks old, male Spraque-Dawley (SD) rats were purchased from Guangdong Medical Laboratory Animal Center (Foshan, China) and maintained in a specific pathogen-free (SPF) animal facility under a $12 \mathrm{~h}$ light $/ 2 \mathrm{~h}$ dark cycle, with free access to food and water. The 5/6 Nx operation was performed in rats under anesthesia with sodium pentobarbital $(50 \mathrm{mg} / \mathrm{kg}$ body weight, intraperitoneal injection) by ablation of upper and lower thirds of the left kidney and then removal of the right kidney 2 weeks later. The sham operation consisting of laparotomy and manipulation of the renal pedicles but without destruction of renal tissue was performed. Twelve weeks after the second surgery, 53 rats remained alive including 10 rats with sham-operated and 43 rats with 5/6 Nx-operated. Thirty-seven $5 / 6 \mathrm{Nx}$ rats with significant higher Scr levels were randomly assigned to 3 groups with 10 rats per group: $5 / 6 \mathrm{Nx}$ (distilled water); $5 / 6 \mathrm{Nx}+$ Perindopril $(4 \mathrm{mg} / \mathrm{kg} / \mathrm{d}) ; 5 / 6 \mathrm{Nx}+\mathrm{JPYSF}(2.72 \mathrm{~g} / \mathrm{kg} / \mathrm{d})$. The same volume of distilled water was given to sham group $(n=$ $10)$. The dosage of perindopril was determined by referring to previous studies $[28,29]$. The dosage of JPYSF was derived from clinical CKD patients and our preliminary experiments. After 6 weeks of treatment, all rats were anesthetized (sodium pentobarbital, $50 \mathrm{mg} / \mathrm{kg}$ body weight, intraperitoneal injection), and blood samples were obtained by cardiac puncture. The rats were euthanized by cervical dislocation without regaining consciousness. Kidneys were removed and preserved for histological analysis, Western blotting, and immunofluorescence analysis.

\section{Biochemical analysis}

Urinary albumin, urinary total protein, urinary $\mathrm{N}$-acetyl- $\beta$-D-glucosaminidase (NAG), urinary creatinine, Scr, BUN, serum albumin, alanine transaminase (ALT), and aspartate transaminase (AST) were measured using a BS-180 automatic biochemistry analyzer (Mindray, Shenzhen, China) following the manufacturer's instructions. 


\section{Histological examination}

Renal pathological injury was evaluated using periodic acid-Schiff (PAS) and Masson's trichrome stains. For quantitative analysis, 40-50 glomerular tuft area and 40-50 proximal tubular lumen cross-sectional area in each rat and five rats in each group were measured using Nikon NIS-Elements BR software version 4.10.00 (Nikon, Japan) to evaluate glomerular hypertrophy and tubular atrophy.

\section{Western blotting}

Equal amounts of kidney cortex lysates were loaded and electrophoresed through 7, 10\%, or 15\% SDS-polyacrylamide gels and were then transferred to nitrocellulose membranes or polyvinylidene difluoride membranes (Millipore, USA). Following blocking in 5\% non-fat milk for $1 \mathrm{~h}$ at room temperature, the membranes were incubated with primary antibodies at $4{ }^{\circ} \mathrm{C}$ overnight. Then, the membranes were incubated in HRP-conjugated secondary antibodies for $1 \mathrm{~h}$ at room temperature. HRP activity was visualized using Clarity Western ECL Substrate and a ChemiDoc MP Imaging System (Bio-Rad Laboratories, USA). Image Lab software version 5.1 was used for densitometric analysis (Bio-Rad Laboratories, USA).

\section{Immunofluorescence analysis}

The paraffin-embedded kidneys were treated by dewaxed, rehydrated, antigen retrieval, and blocking. Then, the sections were stained with primary antibodies at $4{ }^{\circ} \mathrm{C}$ overnight followed by appropriate secondary antibodies. Nuclei were counterstained with the fluorescent dye 4',6-diamidino-2-phenylindole (DAPI). In all cases, antibody negative controls were used to ensure the truth of positive staining. All images were captured by fluorescence microscope (Nikon, Japan).

\section{Statistical analysis}

Data are shown as mean \pm SEM. Statistical significance among groups were tested by one-way ANOVA and post hoc analysis with the Least Significant Difference (LSD) test or the Games-Howell test. $P<0.05$ was considered statistically significant. Data were analyzed using SPSS statistics software (version 16.0, SPSS Inc., Chicago, IL, USA).

\section{Results}

\section{Preparation of standardized JPYSF extract}

In order to chemically standardize JPYSF extract, we established an HPLC-MS method to reveal its HPLC profile and quantify the main components. Eight chemical markers were found in JPYSF extract (Fig. 1a). A typical HPLC-MS profile was developed for JPYSF extract (Fig. 1b), which served as an index for the identification of JPYSF. Moreover, eight chemical markers were quantified in the JPYSF extract defining the minimum requirement for $1 \mathrm{~g}$ of dried powder of standardized JPYSF, i.e., sodium danshensu $(0.45 \mathrm{mg} / \mathrm{g})$; salvianolic acid $\mathrm{B}(1.80 \mathrm{mg} / \mathrm{g})$; echinacoside $(0.50 \mathrm{mg} / \mathrm{g})$; calycosin 7-O- $\beta$-glucoside $\quad(0.68 \mathrm{mg} / \mathrm{g}) ; \quad$ acteoside $\quad(0.10 \mathrm{mg} / \mathrm{g})$; liquiritin $(0.60 \mathrm{mg} / \mathrm{g})$; astragaloside IV $(0.05 \mathrm{mg} / \mathrm{g})$; formononetin $(0.60 \mathrm{mg} / \mathrm{g})$. The yield of the extraction was $\sim 32.59 \pm 1.1 \%(w / w$, Mean \pm SD, $n=3)$. The extract being used here reached the aforesaid requirements, which guaranteed the repeatability of biological results.

\section{Body weight and biochemical profiling}

The timeline of present study was showed in Fig. 2a. Compared with sham group, rats in the $5 / 6 \mathrm{Nx}$ groups showed substantial decline in body weight throughout the experiment. Administration of JPYSF could retard weight loss in 5/6 Nx rats at the final 6 weeks for treatment $(P<0.01)$ (Fig. 2b). Rats in the $5 / 6 \mathrm{Nx}$ group showed lower serum albumin level, higher Scr and BUN levels, which could be restored by perindopril or JPYSF treatment (Fig. 2c-e). In urinary marker respect, the decrease of urinary albumin excretion was not as significant as perindopril treatment in 5/6 Nx + JPYSF group (Fig. 2b). However, both urinary total protein and NAG levels were significantly reduced following treatment of perindopril or JPYSF (Fig. $2 g$ and $h$ ). The levels of serum ALT and AST were not significantly different among four groups (Fig. 2i and j).

JPYSF ameliorated renal pathological injury in $5 / 6 \mathrm{Nx}$ rats PAS-stained sections revealed normal kidney structure in the sham group. In contrast, prominent glomerular hypertrophy and tubular atrophy were observed in the $5 / 6 \mathrm{Nx}$ group, which were further proved by quantitative analyses. Administration of perindopril or JPYSF significantly ameliorated pathological injury occurred in $5 / 6 \mathrm{Nx}$ rats (Fig. 3a-c). Apart from glomerular and tubular injury, rats in the $5 / 6 \mathrm{Nx}$ group also showed obvious interstitial fibrosis observed in Masson-stained sections, which was improved by perindopril or JPYSF treatment (Fig. 3d).

\section{JPYSF down-regulated fibrosis-associated protein expression in $5 / 6 \mathrm{Nx}$ rats}

Western blotting revealed that the expression levels of fibronectin, type IV collagen, and $\alpha$-SMA were all up-regulated in renal cortex of the $5 / 6 \mathrm{Nx}$ group. Administration of perindopril or JPYSF significantly reduced these fibrosis-associated proteins expression (Fig. 4a-d). Immunofluorescence (IF) analysis further proved above results (Fig. 4e). 
a<smiles>CC(=O)C(O)Cc1ccc(O)c(O)c1</smiles>

1: Sodium danshensu

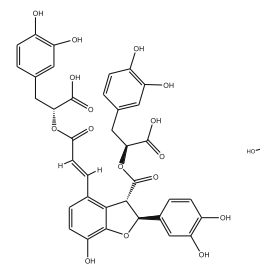

2: Salvianolic acid B<smiles>CCC1CC(CCC2CC(CCC3CCC(C)C3C)C(C(C)C)C2C2C(C)C(C)C(C)C2C)CC1CCC1CC(C)C(C)C1C</smiles>

3: Echinacoside

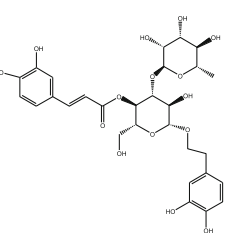

5: Acteoside

6: Calycosin 7-O- $\beta$-glucoside

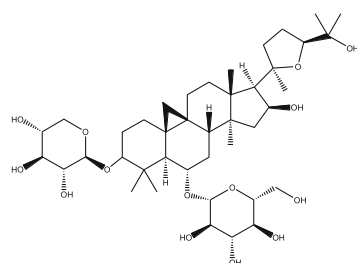

7: Astragaloside IV

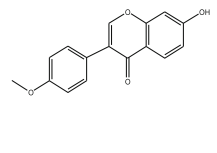

8: Formononetin

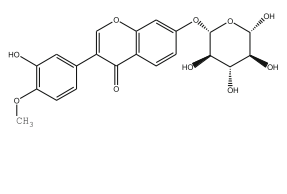

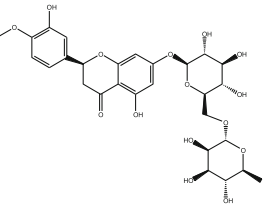

ISTD: Hesperidin

b

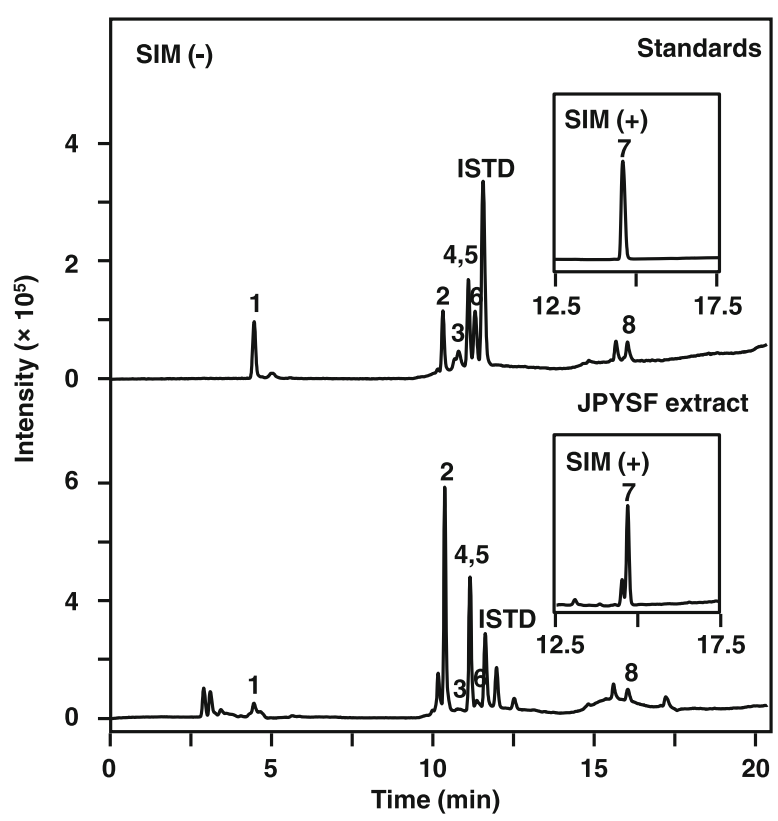

Fig. 1 Typical LC-MS chromatogram of JPYSF. a Structures of chemical markers analyzed in JPYSF extract, including sodium danshensu (1), salvianolic acid B (2), echinacoside (3), liquiritin (4), acteoside (5), calycosin 7-O- $\beta$-glucoside (6), astragaloside IV (7), formononetin (8), and hesperidin (internal standard, ISTD). b The representative LC-MS chromatograms of mixed standards and JPYSF extract. The LC condition was described in the Methods. The denotations from 1 to 8 in the chromatogram correspond to the chemical markers as shown in (a). The identification of the chemical markers was determined by a MS detector in a negative mode except astragaloside IV (7) in a positive mode. Representative chromatograms were shown, $n=3$ 

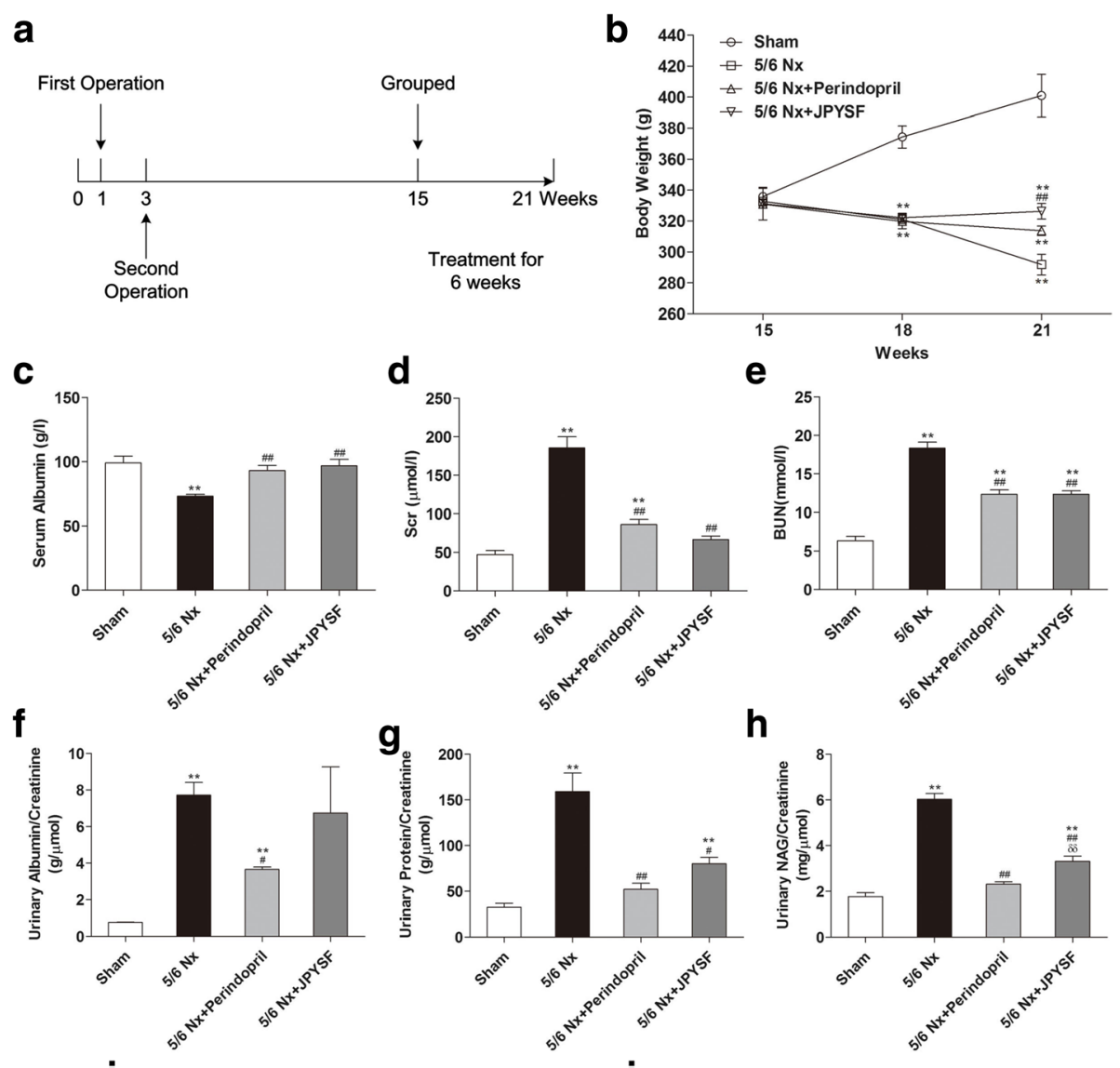

h
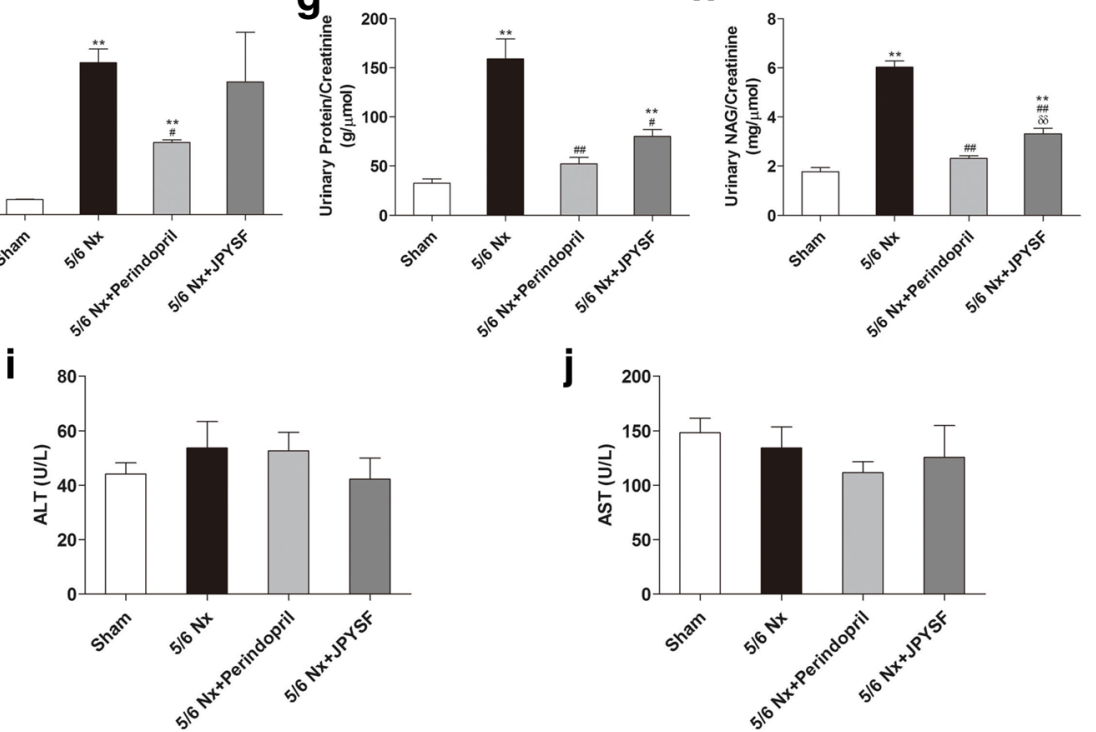

Fig. 2 Body weight and biochemical indexes. a Timeline of study process. b Body weight changes during experiment. c Serum albumin levels. d Scr levels. e BUN levels. $\mathbf{f}$ Urinary albumin excretion adjusted by urinary creatinine. $\mathbf{g}$ Urinary total protein excretion adjusted by urinary creatinine. h Urinary NAG excretion adjusted by urinary creatinine. i Serum ALT levels. j Serum AST levels. Data are presented as the means \pm SEM, $n=6$ rats per group. ( ${ }^{* *} P<0.01$ compared with the sham group; ${ }^{\#} P<0.05,{ }^{\# \#} P<0.01$ compared with the $5 / 6 \mathrm{Nx}$ group; ${ }^{\delta \delta} P<0.01$ compared with the $5 / 6$ $\mathrm{Nx}+$ Perindopril group)

JPYSF up-regulated subunits of mitochondrial respiratory complex in 5/6 Nx rats

Producing adenosine triphosphate (ATP) via respiratory complex is an important function of mitochondrion. As shown in Fig. 5, protein levels of ATP5B, a subunit of mitochondrial ATP synthase, COX-I, COX-IV, and NDUF $\beta 8$, three subunits of mitochondrial respiratory complex, were all reduced in renal cortex of the $5 / 6 \mathrm{Nx}$ group. These proteins expression were all restored in the $5 / 6 \mathrm{Nx}+$ perindopril group and the $5 / 6 \mathrm{Nx}+$ JPYSF group.
JPYSF modulated mitochondrial quality control network in $5 / 6 \mathrm{Nx}$ rats

As shown in Western blot images (Fig. 6a-d), the protein abundance of PGC- $1 \alpha$, the master regulator of mitochondrial biogenesis, and its downstream signal NRF-1 and TFAM were significantly decreased in the $5 / 6 \mathrm{Nx}$ group, whereas these protein levels were significantly increased following treatment of perindopril or JPYSF. The expression of PGC- $1 \alpha$ and TFAM were further proved by IF analysis (Fig. 6e). 


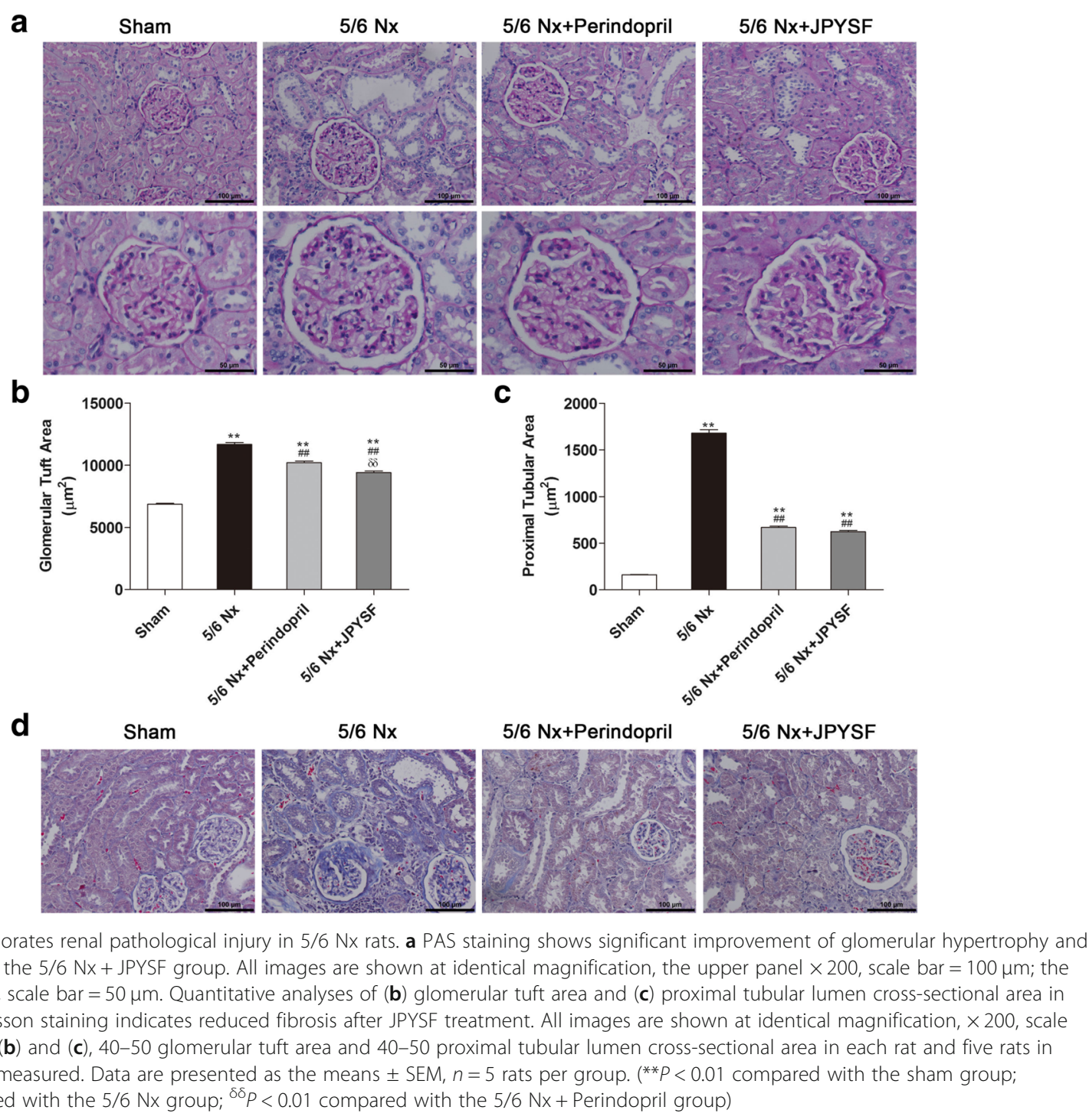

Mitochondrial morphology is governed by the balance between fission and fusion. Rats in the $5 / 6 \mathrm{Nx}$ group presented increased mitochondrial fission and decreased mitochondrial fusion, as evidenced by up-regulation of Drp-1 and down-regulation of Mfn-2 and OPA-1 in Western blot analysis (Fig. 7a-d) and IF analysis (Fig. 7e).

PINK1/Parkin-mediated mitophagy is the best known mitophagy pathway in mammalian cell. PINK1 expression was overtly down-regulated in renal cortex of the $5 / 6 \mathrm{Nx}$ group (Fig. 8a, b). PINK1 stabilization and accumulation in mitochondria would further recruit Parkin from the cytoplasm to the mitochondria and phosphorylate it at Serine 65. Western blotting showed that p-Parkin (Ser 65) was also down-regulated in renal cortex of the $5 / 6 \mathrm{Nx}$ group (Fig. 8a, c). IF analysis indicated that less Parkin colocalized with HSP-60, a mitochondrial marker, in the $5 / 6 \mathrm{Nx}$ group (Fig. 8d). Furthermore, the colocalization of
HSP-60 and LAMP-1, a marker of lysosome, was also less in the $5 / 6 \mathrm{Nx}$ group, compared with the sham group (Fig. 8e). Above data suggested that PINK1/Parkin-mediated mitophagy was down-regulated in the kidney of $5 / 6 \mathrm{Nx}$ rats. Treatment of perindopril or JPYSF significantly restored PINK1/Parkin-mediated mitophagy in 5/6 Nx rats. Taken together, these data suggest that mitochondrial quality control network was disturbed in the kidney of $5 / 6$ $\mathrm{Nx}$ rats, and could be restored by JPYSF treatment.

\section{Discussion}

Accumulated evidence have been indicated that pathological mechanisms are associated with the excessive accumulation of extracellular matrix and podocyte loss and inflammation as well as abnormal lipid metabolism and amino metabolism in CKD patient and animal models [30-34]. However, the status of mitochondrial quality 


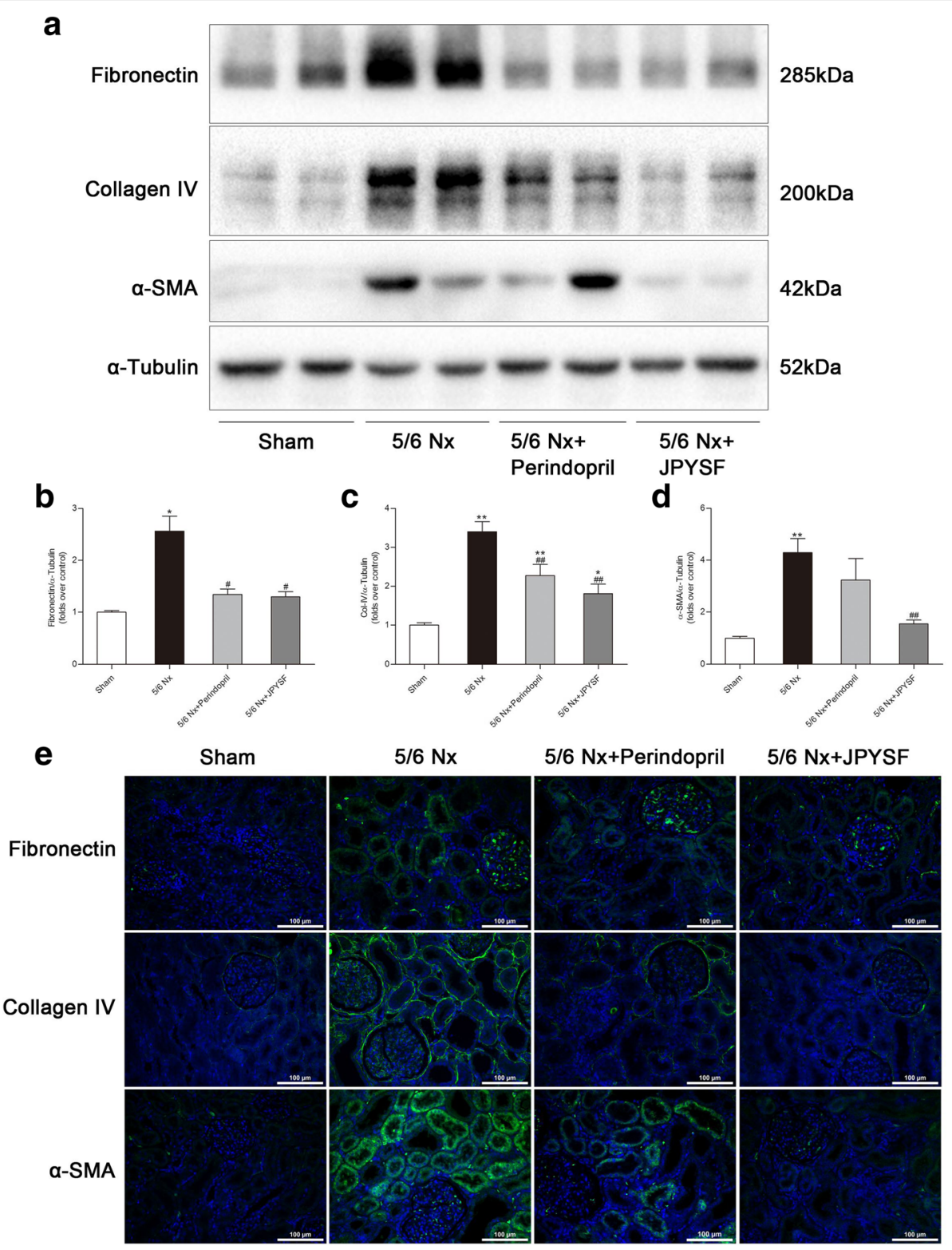

Fig. 4 JPYSF retards renal fibrosis in 5/6 Nx rats. a Representative Western blot images indicate that fibronectin, collagen IV, and a-SMA protein expression were significantly increased in the $5 / 6$ Nx group but were abolished by JPYSF treatment. b-d Densitometric analysis of fibronectin, collagen IV, and a-SMA protein expression normalized to a-tubulin content. e Representative immunofluorescence images of fibronectin, collagen IV, and a-SMA. Green corresponds to interest proteins, and blue corresponds to nuclear staining. All images are shown at identical magnification, $\times 200$, scale bar $=100 \mu \mathrm{m}$. Data are presented as the means \pm SEM, $n=6$ rats per group. $\left({ }^{*} P<0.05,{ }^{* *} P<0.01\right.$ compared with the sham group; ${ }^{\#} P<0.05, \# P<0.01$ compared with the $5 / 6$ Nx group)

control network in CKD was less investigated. In the present study, we successfully reproduced characteristics of CKD in a rat model of $5 / 6 \mathrm{Nx}$, as evidenced by decreased kidney function, proteinuria, and damaged kidney structure. A traditional Chinese herbal formula JPYSF could improve kidney function; ameliorate proteinuria and renal pathological injury. Furthermore, we found obvious mitochondrial dysfunction in 5/6 Nx rats accompanied by disturbed mitochondrial quality control network, which could be restored and modulated by treatment of JPYSF. In the present study, JPYSF and perindopril have comparable effects on improving kidney function. However, our previous studies have demonstrated that JPYSF could induce erythropoietin expression [35], which is related with renal anemia, and improve muscle atrophy in 5/6 Nx rats [36]. Thus, the effect of JPYSF seems to be holistic and multi-target, which are advantages of JPYSF compared with perindopril. 

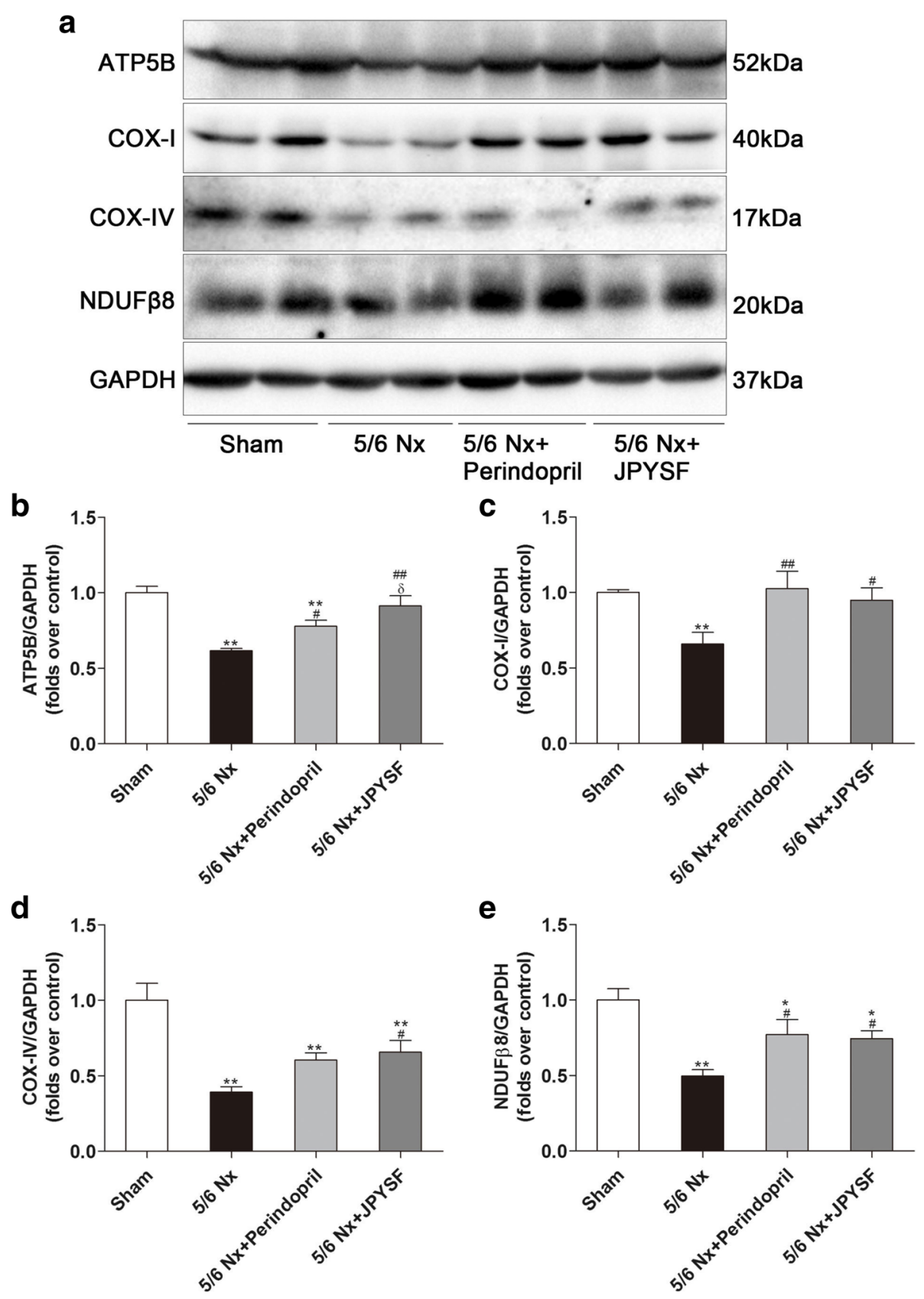

Fig. 5 JPYSF improves mitochondrial function in 5/6 Nx rats. a Representative Western blot images indicate that ATP5B, COX-I, COX-IV, and NDUFB8 protein expression were significantly reduced in the 5/6 Nx group but were increased by JPYSF treatment. $\mathbf{b}$-e Densitometric analysis of ATP5B, COX-I, COX-IV, and NDUFB8 protein expression normalized to GAPDH content. Data are presented as the means \pm SEM, $n=6$ rats per group. ${ }^{*} P<0.05$, ${ }^{* *} P<0.01$ compared with the sham group; ${ }^{\#} P<0.05,{ }^{\# \#} P<0.01$ compared with the $5 / 6 \mathrm{Nx}$ group; ${ }^{\delta} P<0.05$ compared with the $5 / 6 \mathrm{Nx}+$ Perindopril group)

Renal fibrosis is the final common pathway by which earlier stages of CKD progress to end-stage renal disease. Anti-fibrotic therapy is an attractive approach to treat patients with CKD. In our study, administration of JPYSF protected kidneys from fibrotic injury by significantly down-regulating expression of fibronectin, type IV collagen, and $\alpha$-SMA in 5/6 Nx rats. Astragali Radix, the 'sovereign medicinal' in JPYSF, has been previously found to have anti-fibrotic effects through inhibition of the transforming growth factor- $\beta 1$ pathway in several cell types and tissues [37-39]. Astragaloside IV, the active ingredient isolated from Astragali Radix and confirmed in JPYSF extract by HPLC-MS analysis (Fig. 1), also has been reported to ameliorate renal fibrosis in 


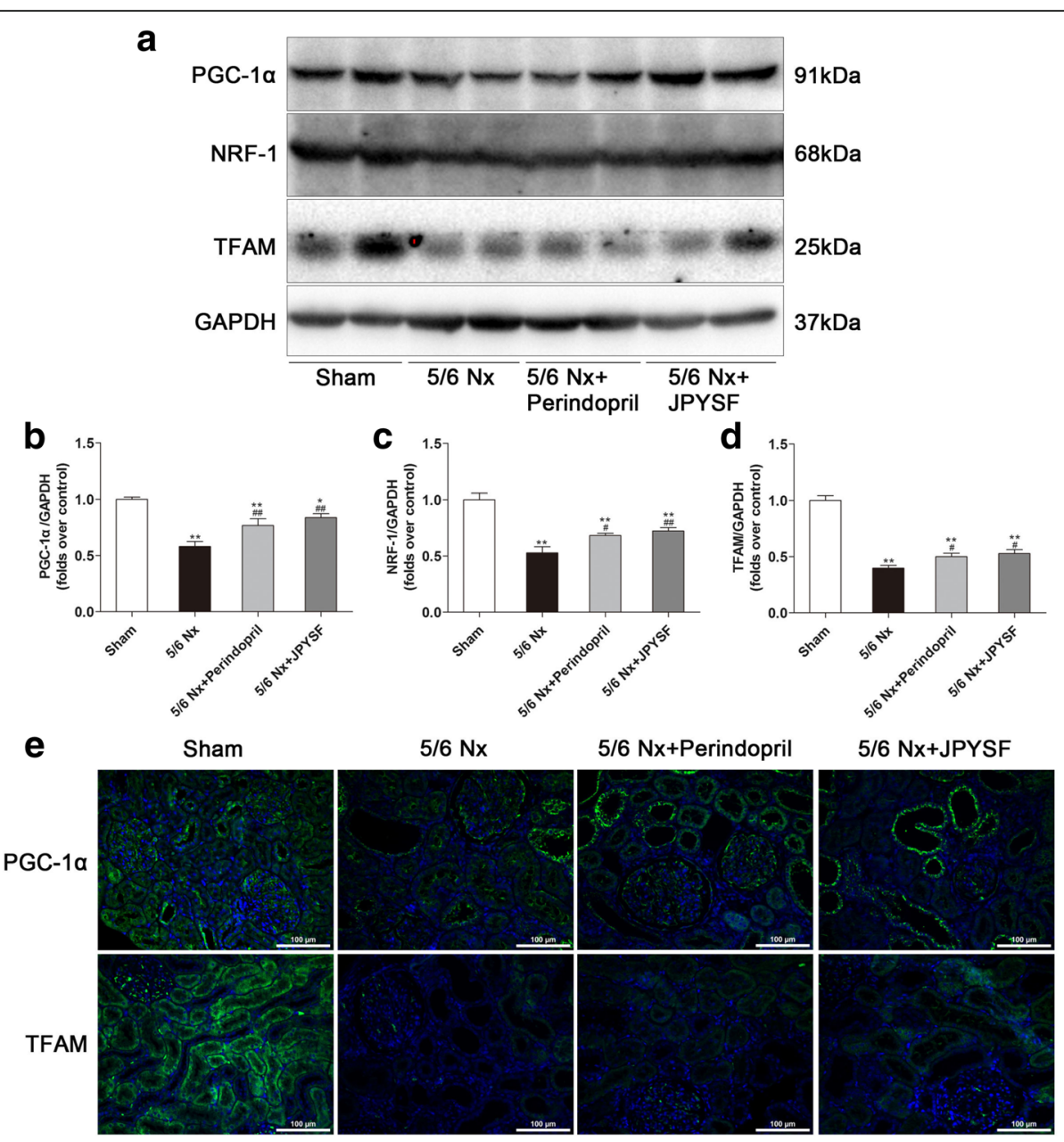

Fig. 6 JPYSF increases mitochondrial biogenesis in 5/6 Nx rats. a Representative Western blot images show decreased PGC-1a, NRF-1, and TFAM expression in the 5/6 Nx group, which were increased by JPYSF treatment. b-d Densitometric analysis of PGC-1a, NRF-1, and TFAM protein expression normalized to GAPDH content. e Representative immunofluorescence images of PGC-1a and TFAM. Green corresponds to interest proteins, and blue corresponds to nuclear staining. All images are shown at identical magnification, $\times 200$, scale bar $=100 \mu \mathrm{m}$. Data are presented as the means \pm SEM, $n=6$ rats per group. $\left({ }^{*} P<0.05,{ }^{* *} P<0.01\right.$ compared with the sham group; ${ }^{*} P<0.05,{ }^{\# \#} P<0.01$ compared with the $5 / 6$ Nx group)

vivo and in vitro [40, 41]. In addition, Salviae Miltiorrhizae Radix et Rhizoma and Rhei Radix et Rhizoma, the 'courier medicinal' of JPYSF, have been observed to decrease levels of kidney extracellular matrix in diabetic $d b / d b$ mice [42], and prevent renal fibrosis by inhibiting epithelial-mesenchymal transition in $\mathrm{HgCl}_{2}$-induced rat model [43]. Apart from three herbs mentioned above, the other components of JPYSF were less reported to have reno-protective effect. However, it is hard to tell which herb most likely conferred the observed therapeutic effects on our CKD model. This may be an orchestrated effect. TGF- $\beta /$ Smad signaling plays pivotal role in the development and progression of renal fibrosis. Previous studies have reported that the main components of JPYSF, including Huang-Qi [44], Dan-Shen [45], and Da-Huang [42], could modulate TGF- $\beta /$ Smad signaling pathway in renal or liver fibrosis. It is speculated that TGF- $\beta /$ Smad signaling may be involved in the anti-fibrosis effect of JPYSF.

Mitochondria are primarily responsible for producing ATP via oxidative phosphorylation in the inner mitochondrial membrane. However, mitochondria can rapidly change into death-promoting organelles by producing excessive reactive oxygen species and releasing prodeath proteins, which will result in disrupted ATP synthesis and activation of cell death pathways [46]. These characteristics of mitochondrion place it in the central position of pathogenesis of metabolic disease, neurodegenerative disease, and cancer [47]. Kidneys are fuel-hungry organs and only second to the heart in mitochondrial number and oxygen consumption [48]. Therefore, mitochondrial dysfunction in the kidneys plays a critical role in the pathogenesis of multiple kidney diseases [18]. In our study, mitochondrial 
a
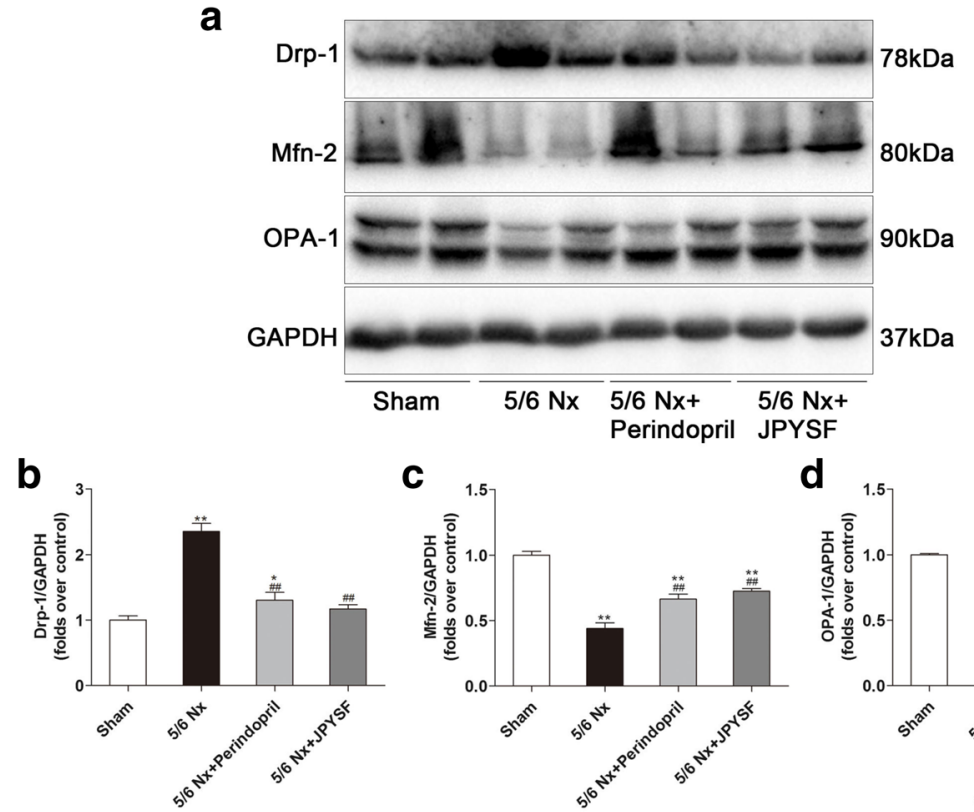

C 1.5

d

e

Sham
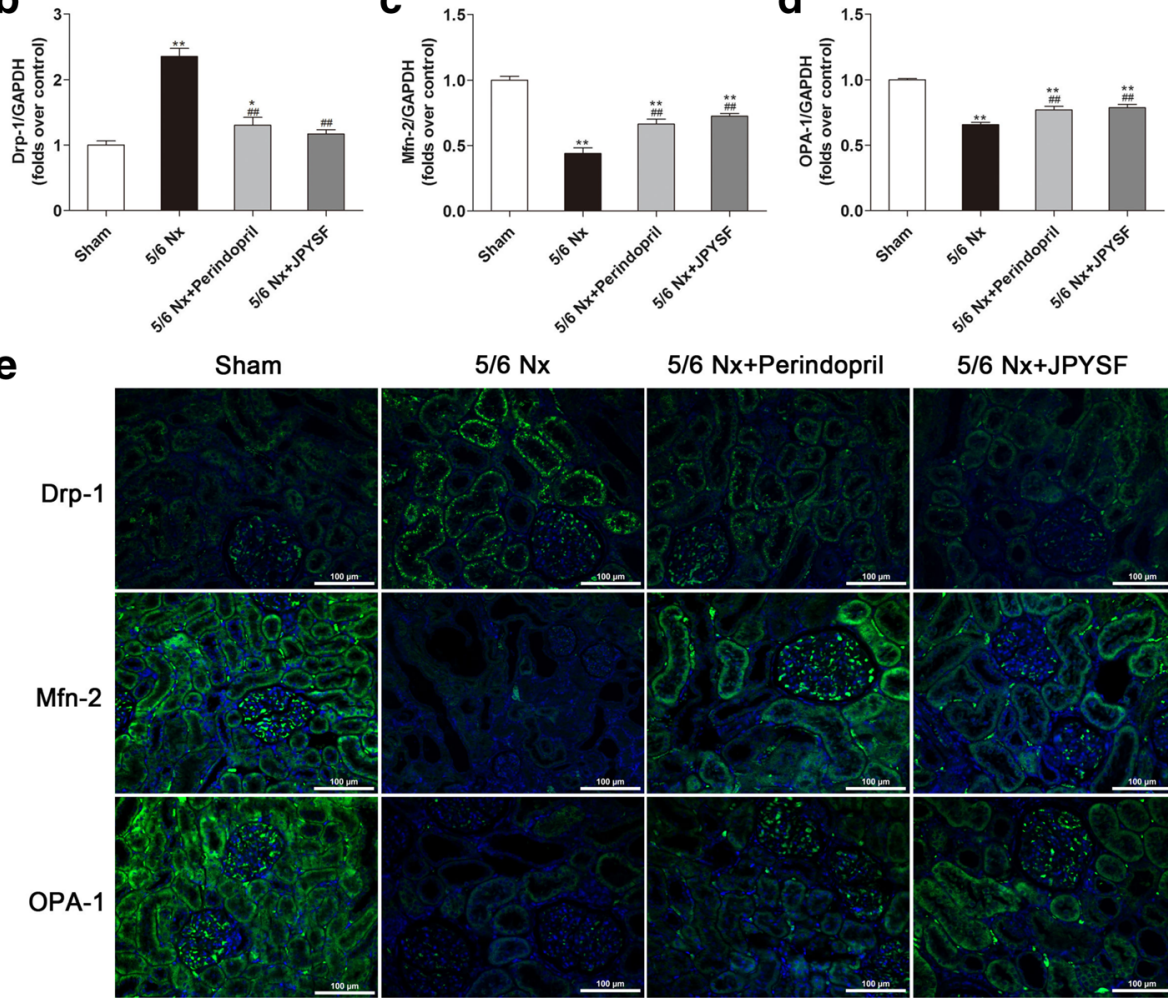

$5 / 6 \mathrm{Nx}$

$5 / 6 \mathrm{Nx}+$ Perindopril

$5 / 6 \mathrm{Nx}+\mathrm{JPYSF}$

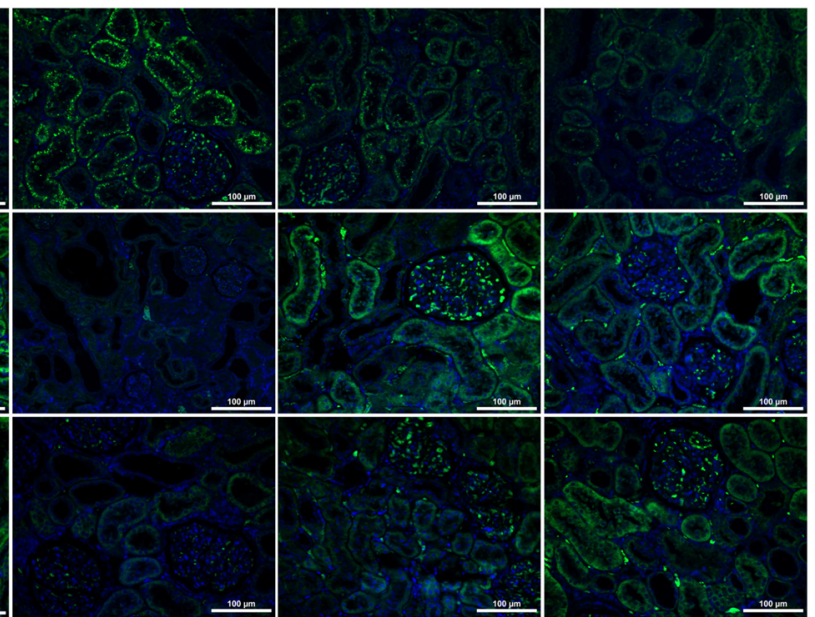

Fig. 7 JPYSF decreases mitochondrial fission and increases mitochondrial fusion. a Representative Western blot images show increased Drp-1, decreased Mfn-2 and OPA-1 expression in the 5/6 Nx group, which were restored by JPYSF treatment. b-d Densitometric analysis of Drp-1, Mfn-2, and OPA-1 protein expression normalized to GAPDH content. e Representative immunofluorescence images of Drp-1, Mfn-2, and OPA-1. Green corresponds to interest proteins, and blue corresponds to nuclear staining. All images are shown at identical magnification, $\times 200$, scale bar $=100 \mu \mathrm{m}$. Data are presented as the means $\pm \mathrm{SEM}, n=6$ rats per group. $\left(^{*} P<0.05,{ }^{*} P<0.01\right.$ compared with the sham group; ${ }^{\# \#} P<0.01$ compared with the $5 / 6 \mathrm{Nx}$ group)

respiratory complex subunits and ATP synthase subunit were all downregulated in 5/6 Nx rats, which indicated disturbed mitochondrial function in CKD. Similar with our results, another study found that COX-IV level was significantly reduced in rat kidneys after 5/6 $\mathrm{Nx}$ insult [49]. More significantly, we found obvious derangement of mitochondrial quality control network in 5/6 Nx rats, presenting as decreased mitochondrial biogenesis, increased mitochondrial fission, decreased mitochondrial fusion, and decreased mitophagy. Mitochondrial quality control network is responsible for mitochondrial homeostasis and mitochondrial function [27]. Our results showed that JPYSF could restore the disturbed protein expression associated with mitochondrial quality control network in $5 / 6 \mathrm{Nx}$ rats, which may be contribute to its effect in improving mitochondrial function. Wallace suggests that mitochondria may be considered as Qi (Chi) [50], which loosely translates as vital force or energy, according to its TCM interpretation. Astragali Radix, the 'sovereign medicinal' in JPYSF, is one of the most important drugs for 'replenishing vital energy' in TCM. And our unpublished data showed that 


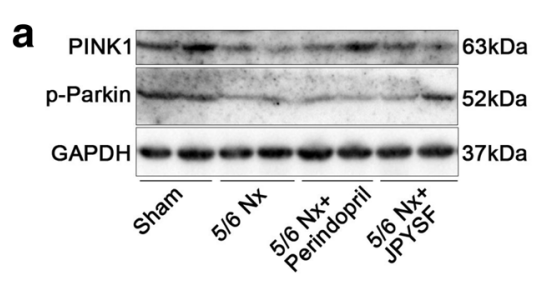

d
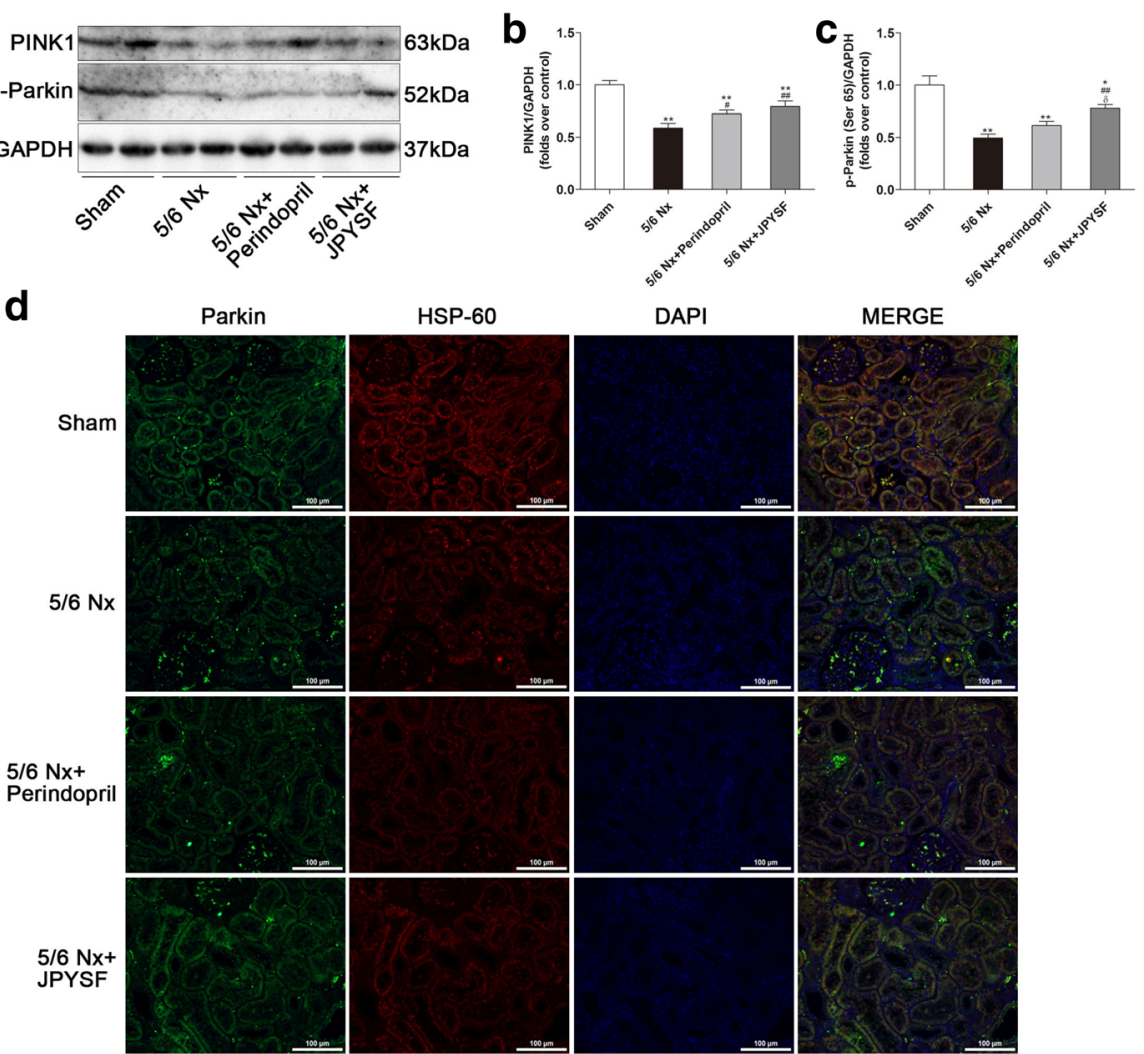

DAPI

MERGE
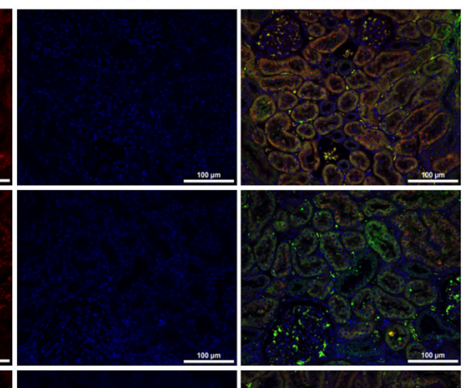

e

LAMP-1

HSP-60

DAPI

MERGE

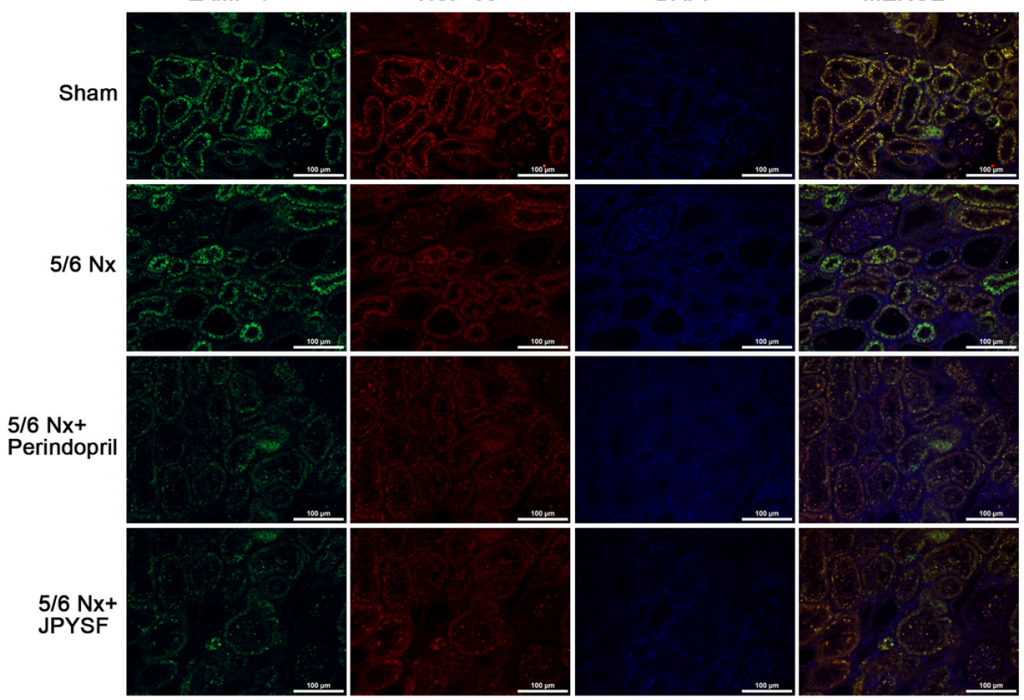

Fig. 8 JPYSF restores the level of mitophagy in 5/6 Nx rats. a Representative Western blot images show decreased PINK1 and p-Parkin expression in the $5 / 6$ Nx group, which were restored by JPYSF treatment. b, c Densitometric analysis of PINK1 and p-Parkin protein expression normalized to GAPDH content. d Representative immunofluorescence images indicating the colocalization of Parkin and HSP-60. e Representative immunofluorescence images indicating the colocalization of LAMP-1 and HSP-60. Green corresponds to interest proteins, red corresponds to mitochondria, and blue corresponds to nuclear staining. All images are shown at identical magnification, $\times 200$, scale bar $=100 \mu m$. Data are presented as the means $\pm \mathrm{SEM}, n=6$ rats per group. $\left({ }^{*} P<0.05\right.$, ${ }^{* *} P<0.01$ compared with the sham group; ${ }^{\#} P<0.05$, ${ }^{\# \#} P<0.01$ compared with the $5 / 6 \mathrm{Nx}$ group; ${ }^{\delta} \mathrm{P}<0.05$ compared with the $5 / 6 \mathrm{Nx}+$ Perindopril group) 
astragaloside IV, the mainly active component of Astragali Radix, could reduce mitochondrial fission in diabetic $d b / d b$ mice. Astragaloside IV has also been reported to increase PGC- $1 \alpha$ expression in vascular smooth muscle cells [51] and rat heart [52]. Thus, it is possible that Astragali Radix in JPYSF most likely affected mitochondrial quality control. Previous studies demonstrated that astragaloside IV [53] and salvianolic acid B [54] could modulate mammalian target of rapamycin (mTOR) signaling pathway, which is close related with mitochondrial quality control. Further study is needed to explore the underlying mechanism of JPYSF in regulating mitochondrial quality control. Collectively, our study shed lights on the regulative effect of TCM formula on mitochondrial quality control network in CKD, which echoes a previous review demonstrated the role of TCM in cardiovascular disease by regulating the structure and function of mitochondria [55]. However, more rigorous pharmacologic studies and detailed mechanistic studies using modern scientific methodology and approaches are needed to elucidate the therapeutic potential of TCM for CKD.

\section{Conclusions}

In conclusion, the present study demonstrates that orally administered JPYSF significantly retards development and progression of CKD in a 5/6 Nx rat model. Our results also suggest that modulating mitochondrial quality control network may be related with the beneficial effect of JPYSF on CKD.

\begin{abstract}
Abbreviations
5/6 Nx: 5/6 nephrectomy; ALT: alanine transaminase; AST: aspartate transaminase; ATP: adenosine triphosphate; ATP5B: ATP synthase subunit beta; BUN: blood urea nitrogen; CKD: chronic kidney disease; COXI: cytochrome c oxidase subunit l; COX-IV: cytochrome c oxidase subunit IV; DAPI: 4',6-diamidino-2-phenylindole; DHGCT: Da-Huang-Gan-Cao-Tang; Drp1: dynamin-related protein 1; GAPDH: glyceraldehyde-3-phosphate dehydrogenase; HRP: horseradish peroxidase; HSP-60: heat shock protein-60; JPYSF: Jian-Pi-Yi-Shen Formula; LAMP-1: Iysosomal-associated membrane protein 1; Mfn-2: mitofusin 2; NAG: N-acetyl- $\beta$-D-glucosaminidase; NDUF $\beta 8$ : nicotinamide adenine dinucleotide dehydrogenase (ubiquinone)-1 $\beta$ subcomplex 8; NRF-1: nuclear respiratory factor 1; OPA-1: optic atrophy 1; PAS: periodic acid-Schiff; PGC-1a: peroxisome proliferator-activated receptorY coactivator-1a; PINK1: PTEN-induced putative kinase 1; TCM: traditional Chinese medicine; TFAM: mitochondrial transcription factor A; YPFS: Yu-PingFeng-San; Scrserum creatinine; a-SMA: a-smooth muscle actin
\end{abstract}

\section{Acknowledgements}

The authors thank Dr. Xuewen Yu for his assistance in pathological staining and analysis.

\section{Funding}

This study was supported by Shenzhen Science and Technology Plan Project (JSGG20141017103353178, JCYJ20160428182041577,

ZDSYS201606081515458, and JCYJ20170307154652899), Natural Science Foundation of Guangdong Province (2015A030310247, 2015A030310252, and 2018A030313305), and Natural Science Foundation of China (81603437 and 81804052). The funders have no role in the design of the study and collection, analysis, and interpretation of data and in writing the manuscript.
Availability of data and materials

The datasets used and/or analysed during the current study are available from the corresponding author on reasonable request.

\section{Authors' contributions}

TGY and SML contributed to conception and design, and modification of the manuscript. XYL and PZ contributed to herbal preparation and acquisition of data. DTW and JPC contributed to animal experiments and pathological analysis. ARQ contributed to acquisition of data and manuscript writing. XHL and JPC contributed to laboratory experiments, analysis and interpretation of data, and manuscript writing. All authors read and approved the final manuscript.

\section{Ethics approval and consent to participate}

All animal experiments were conducted with protocols approved by the Ethics Committee of Guangzhou University of Chinese Medicine and in accordance with National Institutes of Health Guideline for the care and use of laboratory animals (NIH Publications No. 80-23, revised 1996).

\section{Consent for publication}

Not applicable.

\section{Competing interests}

The authors declare that they have no competing interests.

\section{Publisher's Note}

Springer Nature remains neutral with regard to jurisdictional claims in published maps and institutional affiliations.

\section{Author details}

${ }^{1}$ Department of Nephrology, Shenzhen Traditional Chinese Medicine Hospital, Guangzhou University of Chinese Medicine, Shenzhen, Guangdong, China. ${ }^{2}$ Shenzhen Key Laboratory of Hospital Chinese Medicine Preparation, Shenzhen Traditional Chinese Medicine Hospital, Guangzhou University of Chinese Medicine, Shenzhen, Guangdong, China.

Received: 14 March 2018 Accepted: 28 November 2018

Published online: 20 December 2018

References

1. Jha V, Garcia-Garcia G, Iseki K, Li Z, Naicker S, Plattner B, et al. Chronic kidney disease: global dimension and perspectives. Lancet. 2013;382:260-72.

2. de Zeeuw D. Unmet need in renal protection--do we need a more comprehensive approach? Contrib Nephrol. 2011;171:157-60.

3. Chen L, Chen DQ, Wang M, Liu D, Chen H, Dou F, et al. Role of RAS/ Wnt/beta-catenin axis activation in the pathogenesis of podocyte injury and tubulo-interstitial nephropathy. Chem Biol Interact. 2017; 273:56-72.

4. Zhong Y, Deng Y, Chen Y, Chuang PY, Cijiang HJ. Therapeutic use of traditional Chinese herbal medications for chronic kidney diseases. Kidney Int. 2013;84:1108-18.

5. Zhao YY. Traditional uses, phytochemistry, pharmacology, pharmacokinetics and quality control of Polyporus umbellatus (Pers.) fries: a review. J Ethnopharmacol. 2013;149:35-48

6. Tian T, Chen H, Zhao YY. Traditional uses, phytochemistry, pharmacology, toxicology and quality control of Alisma orientale (Sam.) Juzep: a review. Ethnopharmacol 2014; 158 Pt A: 373-387.

7. Zhang $Z H$, Vaziri ND, Wei F, Cheng XL, Bai $X$, Zhao YY. An integrated lipidomics and metabolomics reveal nephroprotective effect and biochemical mechanism of Rheum officinale in chronic renal failure. Sci Rep. 2016;6:22151.

8. Zhao YY, Chen H, Tian T, Chen DQ, Bai X, Wei F. A pharmaco-metabonomic study on chronic kidney disease and therapeutic effect of ergone by UPLCQTOF/HDMS. PLoS One. 2014;9:e115467.

9. Zhang L, Wang F, Wang L, Wang W, Liu B, Liu J, et al. Prevalence of chronic kidney disease in China: a cross-sectional survey. Lancet. 2012;379:815-22.

10. Zhong Y, Menon MC, Deng Y, Chen Y, He JC. Recent advances in traditional Chinese medicine for kidney disease. Am J Kidney Dis. 2015;66:513-22.

11. Zhang ZH, Wei F, Vaziri ND, Cheng XL, Bai X, Lin RC, et al. Metabolomics insights into chronic kidney disease and modulatory effect of rhubarb against tubulointerstitial fibrosis. Sci Rep. 2015;5:14472. 
12. Zhao YY, Zhang L, Mao JR, Cheng XH, Lin RC, Zhang Y, et al. Ergosta4,6,8(14),22-tetraen-3-one isolated from Polyporus umbellatus prevents early renal injury in aristolochic acid-induced nephropathy rats. J Pharm Pharmacol. 2011;63:1581-6.

13. Zhao YY, Feng YL, Bai X, Tan XJ, Lin RC, Mei Q. Ultra performance liquid chromatography-based metabonomic study of therapeutic effect of the surface layer of Poria cocos on adenine-induced chronic kidney disease provides new insight into anti-fibrosis mechanism. PLoS One. 2013;8:e59617.

14. Lin MY, Chiu YW, Chang JS, Lin HL, Lee CT, Chiu GF, et al. Association of prescribed Chinese herbal medicine use with risk of end-stage renal disease in patients with chronic kidney disease. Kidney Int. 2015;88:1365-73.

15. Yang D, Yang SD, Li SM. Introduction of three kidney diseases treated from the spleen by professor Li Shunmin. World Chin Med. 2015;10:831-3 [in Chinese].

16. Yi TG, Qi AR, Yi WY. The clinical study of the Jianpiyishenfang in treating chronic renal failure. Chin J Integr Tradit West Nephrol. 2004;5:149-51 [in Chinese].

17. Li SM, Fu B, Yi TG, Yi WY, Yang SD, Yang D, et al. Research on chronic renal failure and malnutrition treated with Jianpi Yishen decoction (method). Chin Arch Tradit Chin Med 2008; 26: 2102-2104. [in Chinese].

18. Che R, Yuan Y, Huang S, Zhang A. Mitochondrial dysfunction in the pathophysiology of renal diseases. Am J Physiol Renal Physiol. 2014;306: F367-78.

19. Granata S, Zaza G, Simone S, Villani G, Latorre D, Pontrelli P, et al. Mitochondrial dysregulation and oxidative stress in patients with chronic kidney disease. BMC Genomics. 2009;10:388

20. Small DM, Coombes JS, Bennett N, Johnson DW, Gobe GC. Oxidative stress, anti-oxidant therapies and chronic kidney disease. Nephrology (Carlton). 2012:17:311-21.

21. Gucer S, Talim B, Asan E, Korkusuz P, Ozen S, Unal S, et al. Focal segmental glomerulosclerosis associated with mitochondrial cytopathy: report of two cases with special emphasis on podocytes. Pediatr Dev Pathol. 2005:8:710-7.

22. Su M, Dhoopun AR, Yuan Y, Huang S, Zhu C, Ding G, et al. Mitochondrial dysfunction is an early event in aldosterone-induced podocyte injury. Am J Physiol Renal Physiol. 2013;305:F520-31.

23. Mutsaers HA, Wilmer MJ, Reijnders D, Jansen J, van den Broek PH, Forkink $M$, et al. Uremic toxins inhibit renal metabolic capacity through interference with glucuronidation and mitochondrial respiration. Biochim Biophys Acta. 2013;1832:142-50.

24. Gewin L, Zent R, Pozzi A. Progression of chronic kidney disease: too much cellular talk causes damage. Kidney Int. 2017;91:552-60.

25. Gong W, Mao S, Yu J, Song J, Jia Z, Huang S, et al. NLRP3 deletion protects against renal fibrosis and attenuates mitochondrial abnormality in mouse with 5/6 nephrectomy. Am J Physiol Renal Physiol. 2016;310:F1081-8.

26. Casalena G, Daehn I, Bottinger E. Transforming growth factor-beta, bioenergetics, and mitochondria in renal disease. Semin Nephrol. 2012;32: 295-303.

27. Fischer F, Hamann A, Osiewacz HD. Mitochondrial quality control: an integrated network of pathways. Trends Biochem Sci. 2012;37:284-92.

28. Komatsu Y, Kida N, Nozaki N, Kuwasako K, Nagata S, Kitamura K, et al. Effects of proangiotensin-12 infused continuously over 14 days in conscious rats. Eur J Pharmacol. 2012;683:186-9.

29. Yuan L, Li X, Xu GL, Qi CJ. Effects of renin-angiotensin system blockade on islet function in diabetic rats. J Endocrinol Investig. 2010;33:13-9.

30. Wang $M$, Chen L, Liu D, Chen H, Tang DD, Zhao YY. Metabolomics highlights pharmacological bioactivity and biochemical mechanism of traditional Chinese medicine. Chem Biol Interact. 2017:273:133-41.

31. Chen DQ, Cao G, Chen H, Liu D, Su W, Yu XY, et al. Gene and protein expressions and metabolomics exhibit activated redox signaling and wnt/ beta-catenin pathway are associated with metabolite dysfunction in patients with chronic kidney disease. Redox Biol. 2017;12:505-21.

32. Chen H, Cao G, Chen DQ, Wang M, Vaziri ND, Zhang ZH, et al. Metabolomics insights into activated redox signaling and lipid metabolism dysfunction in chronic kidney disease progression. Redox Biol. 2016;10:168-78.

33. Chen DQ, Chen H, Chen L, Vaziri ND, Wang M, Li XR, et al. The link between phenotype and fatty acid metabolism in advanced chronic kidney disease. Nephrol Dial Transplant. 2017;32:1154-66.

34. Chen $\mathrm{H}$, Chen L, Liu D, Chen DQ, Vaziri ND, Yu XY, et al. Combined clinical phenotype and Lipidomic analysis reveals the impact of chronic kidney disease on lipid metabolism. J Proteome Res. 2017:16:1566-78.
35. Chen J, Gong AGW, Liu X, Li Z, Qi A, Dong TTX, et al. A Chinese herbal decoction, Jian-Pi-Yi-Shen, regulates the expressions of erythropoietin and pro-inflammatory cytokines in cultured cells. BMC Complement Altern Med. 2018:18:119.

36. Wang D, Chen J, Liu X, Zheng P, Song G, Yi T, et al. A Chinese herbal formula, Jian-Pi-Yi-Shen decoction, improves muscle atrophy via regulating mitochondrial quality control process in 5/6 nephrectomised rats. Sci Rep. 2017;7:9253.

37. Huang W, Li L, Tian X, Yan J, Yang X, Wang X, et al. Astragalus and Paeoniae radix rubra extract inhibits liver fibrosis by modulating the transforming growth factorbeta/Smad pathway in rats. Mol Med Rep. 2015;11:805-14.

38. Li Z, Zhang L, He W, Zhu C, Yang J, Sheng M. Astragalus membranaceus inhibits peritoneal fibrosis via monocyte chemoattractant protein (MCP)-1 and the transforming growth factor-beta1 (TGF-beta1) pathway in rats submitted to peritoneal dialysis. Int J Mol Sci. 2014:15:12959-71.

39. Gu J, Liu K, Li H, Wang X, Yang K. Astragalus saponin attenuates the expression of fibrosis-related molecules in irradiated cardiac fibroblasts. Acta Biochim Biophys Sin Shanghai. 2014;46:492-501.

40. Xu W, Shao X, Tian L, Gu L, Zhang M, Wang Q, et al. Astragaloside IV ameliorates renal fibrosis via the inhibition of mitogen-activated protein kinases and antiapoptosis in vivo and in vitro. J Pharmacol Exp Ther. 2014; 350:552-62.

41. Wang L, Chi YF, Yuan ZT, Zhou WC, Yin PH, Zhang XM, et al. Astragaloside IV inhibits renal tubulointerstitial fibrosis by blocking TGF-beta/Smad signaling pathway in vivo and in vitro. Exp Biol Med (Maywood). 2014;239: 1310-24.

42. Gao Q, Qin WS, Jia ZH, Zheng JM, Zeng CH, Li LS, et al. Rhein improves renal lesion and ameliorates dyslipidemia in $\mathrm{db} / \mathrm{db}$ mice with diabetic nephropathy. Planta Med. 2010;76:27-33.

43. Wang QL, Tao YY, Yuan JL, Shen L, Liu CH. Salvianolic acid B prevents epithelial-to-mesenchymal transition through the TGF-beta1 signal transduction pathway in vivo and in vitro. BMC Cell Biol. 2010;11:31.

44. Zhao J, Wang L, Cao AL, Jiang MQ, Chen X, Wang Y, et al. HuangQi decoction ameliorates renal fibrosis via TGF- $\beta /$ Smad signaling pathway in vivo and in vitro. Cell Physiol Biochem. 2016:38:1761-74.

45. Yin D, Yin J, Yang Y, Chen S, Gao X. Renoprotection of Danshen injection on streptozotocin-induced diabetic rats, associated with tubular function and structure. J Ethnopharmacol. 2014;151:667-74.

46. Galluzzi L, Kepp O, Trojel-Hansen C, Kroemer G. Mitochondrial control of cellular life, stress, and death. Circ Res. 2012;111:1198-207.

47. Chandel NS. Evolution of mitochondria as signaling organelles. Cell Metab. 2015;22:204-6.

48. Forbes JM. Mitochondria-power players in kidney function? Trends Endocrinol Metab. 2016;27:441-2

49. Fedorova LV, Tamirisa A, Kennedy DJ, Haller ST, Budnyy G, Shapiro Jl, et al. Mitochondrial impairment in the five-sixth nephrectomy model of chronic renal failure: proteomic approach. BMC Nephrol. 2013;14:209.

50. Wallace DC. Mitochondria as chi. Genetics. 2008:179:727-35.

51. Lu Y, Li S, Wu H, Bian Z, Xu J, Gu C, et al. Beneficial effects of astragaloside IV against angiotensin II-induced mitochondrial dysfunction in rat vascular smooth muscle cells. Int J Mol Med. 2015;36:1223-32.

52. Zhang S, Tang F, Yang Y, Lu M, Luan A, Zhang J, et al. Astragaloside IV protects against isoproterenol-induced cardiac hypertrophy by regulating NF-kappaB/PGC-1alpha signaling mediated energy biosynthesis. PLoS One. 2015:10:e118759.

53. Sun $\mathrm{H}$, Wang $W$, Han $P$, Shao $M$, Song $G$, Du H, et al. Astragaloside IV ameliorates renal injury in db/db mice. Sci Rep. 2016;6:32545.

54. Gong L, Di C, Xia X, Wang J, Chen G, Shi J, et al. AKT/mTOR signaling pathway is involved in salvianolic acid B-induced autophagy and apoptosis in hepatocellular carcinoma cells. Int J Oncol. 2016;49:2538-48.

55. Wang J, Lin F, Guo LL, Xiong XJ, Fan X. Cardiovascular disease, mitochondria, and traditional Chinese medicine. Evid Based Complement Alternat Med. 2015;2015:143145. 